\title{
Overexpression of fatty acid synthase predicts a poor prognosis for human gastric cancer
}

\author{
JIANGMAN DUAN, LI SUN, HONGXIANG HUANG, ZHENZHEN WU, LIN WANG and WANGJUN LIAO \\ Department of Oncology, Nanfang Hospital, Southern Medical University, Guangzhou, Guangdong 510515, P.R. China
}

Received January 21, 2015; Accepted November 6, 2015

DOI: $10.3892 / \mathrm{mmr} .2016 .4902$

\begin{abstract}
Fatty acid synthase (FASN), a lipogenic multi-enzyme complex, is reported to be overexpressed in various types of of tumor tissues and serves an important role in tumor development and progression. However, the expression of FASN and its possible role in gastric cancer (GC) remains to be defined. In the present study, FASN expression in a group sample of $167 \mathrm{GC}$ tissues was detected by immunohistochemistry and its correlation with clinicopathological features was analyzed. By clinical analysis, it was identified that FASN overexpression was positively correlated with the overall survival $[\mathrm{P}=0.008$; hazard ratio $(\mathrm{HR}), 4.412$; 95\% confidence interval (CI), 1.463-13.305] and recurrence rate $(\mathrm{P}=0.014$; HR, $1.705 ; 95 \% \mathrm{CI}, 1.116-2.606)$ in patients with GC. In addition, expression of the FASN protein in GC tissues was correlated with age $(\mathrm{P}=0.032)$, clinical stage $(\mathrm{P}<0.001)$, gastric wall invasion $(\mathrm{P}=0.014)$, lymph node metastasis $(\mathrm{P}<0.001)$ and distant metastasis $(\mathrm{P}<0.001)$, however not with gender $(\mathrm{P}>0.05)$. In addition, FASN was observed to be overexpressed in GC tissues at an mRNA and protein level, compared with the adjacent non-cancerous tissues $(\mathrm{P}<0.05)$. Taken together, it was suggested that FASN was closely associated with GC metastasis and survival, which further provided evidence that FASN may be a promising prognostic biomarker for patients with $\mathrm{GC}$.
\end{abstract}

\section{Introduction}

Gastric cancer (GC) is the second leading cause of global cancer-associated mortality with a notably high incidence in developing countries, with approximately 692,720 newly diagnosed cases and 516,600 cases of GC-associated mortality in 2011 (1-3). Greater than $60 \%$ patients with GC were diagnosed

Correspondence to: Professor Wangjun Liao, Department of Oncology, Nanfang Hospital, Southern Medical University, 1838 North Guangzhou Avenue, Guangzhou, Guangdong 510515, P.R. China

E-mail: nfyyliaowj@163.com

Key words: gastric cancer, fatty acid synthase, prognosis, lipogenesis, metabolism at an advanced stage with locally advanced or distant metastasis, which leads to a poorer prognosis and relatively increased mortality $(4,5)$. Accordingly, early detection of GC will contribute to a reduction in its mortality. Numerous genes have been associated with the prognosis of GC; however, aside from Her-2, few sensitive biomarkers have been reported in clinical applications (6). In addition, a previous study demonstrated that only $17 \%$ of patients with GC are associated with a HER-2-positive state (7). Therefore, it is of great clinical significance to identify specific and sensitive biomarkers for GC early detection and effective therapeutic targets.

Several in vitro studies have indicated that elevated lipogenesis is correlated with poor prognosis in a number of tumor types (8-11). In addition, lipogenesis has been demonstrated to be involved in signal transduction of tumor cells (12-14). As a key cytosolic multifunctional enzyme involved in de novo lipogenesis, fatty acid synthase (FASN) is overexpressed in several types of tumor tissue and is significantly associated with tumor prognosis $(15,16)$. In addition, reduction of FASN activity markedly promotes tumor apoptosis and inhibits tumor cell growth and metastasis (17-20).

However, studies focussing upon FASN in GC are rare. Two previous studies have provided evidence that FASN is overexpressed in GC tissues (21) in addition to blood serum (22). FASN overexpression is associated with poor survival of patients with GC, indicating that FASN serves a crucial role in the development and progression of GC. However, the specific pro-tumor effects of FASN, particularly the detailed correlation of FASN expression and clinicopathological characteristics, remain unclear in GC. Thus, in the present study, immunohistochemistry (IHC), reverse transcription-quantitative polymerase chain reaction (RT-qPCR) and western blotting were conducted in order to analyze the expression levels of FASN in a total of 182 clinical gastric specimens (167 for IHC, 12 for RT-qPCR and 3 for western blotting). In addition, the detailed association between FASN expression and GC clinicopathological characteristics, and clinical prognosis, were investigated further.

\section{Materials and methods}

Patients and tissue specimens. The present study was approved by the Ethics Review Board of Nanfang Hospital (Guangzhou, China), and written informed consent was obtained from all patients. The present study was conducted 
on tissue specimens from 167 patients who had been histologically diagnosed as having GC at Nanfang Hospital between 2000 and 2011, and tumor staging was defined according to the American Joint Committee on Cancer Staging Manual (23). Among them, 131 stage I-III patients received radical resection (19, 49 and 63 for stages I, II and III, respectively), and 36 stage-IV patients (the metastasis-affected distant organs) underwent palliative surgery and/or chemotherapy. Postoperative follow-up time was obtained from all patients from 0.5 to 80.0 months. A total of 12-paired tumor and corresponding normal gastric tissues were rapidly removed during surgery and stored immediately at $-80^{\circ} \mathrm{C}$ until required for RNA extraction, and 3-paired tissues were used for protein extraction.

IHC assays. IHC assays were conducted in order to evaluate the expression of FASN in gastric tissue samples according to the standard protocols. Specimens were paraffin-embedded (Shanghai Specimen and Model Factory, Shanghai, China) and then stored at $4^{\circ} \mathrm{C}$. The paraffin-embedded sections were deparaffinized with xylene (Guangzhou Chemical Reagent Factory, Guangzhou, China) graded ethanol, and phosphate-buffered saline (PBS). Subsequent to quenching the endogenous peroxidase activity with $3 \%$ hydrogen peroxide (Hengjian Pharmaceutical Co., Ltd., Guangzhou, China) for $10 \mathrm{~min}$ at room temperature, the primary rabbit anti-human FASN polyclonal antibody (1:200; 3180S; Cell Signaling Technology, Inc., Danvers, MA, USA) was added and incubated at $4^{\circ} \mathrm{C}$ overnight. Subsequent to washing with PBS, the sections were incubated with horseradish peroxidase (HRP)-conjugated goat anti-rabbit IgG (PV-6001; LI-COR, Inc., Lincoln, NE, USA) for $1 \mathrm{~h}$ at $37^{\circ} \mathrm{C}$. Antibody binding was visualized by incubating with fresh $3,3^{\prime} \mathrm{N}$-diaminobenzidine (Dako, Glostrup, Denmark) buffer. The sections were then washed in running water and counterstained with hematoxylin (Guangzhou Chemical Reagent Factory), followed by dehydration using graded ethanol and mounting using neutral balsam (Shanghai Specimen and Model Factory). Images of the sections were then captured under the Olympus BX40 microscope (Olympus Corporation, Tokyo, Japan).

The FASN expression level in tumor tissues was scored using a semi-quantitative method (19). The intensity of the immunostaining was scored as follows: 0 (negative), 1 (weak), 2 (moderate) and 3 (strong). The extent of immunostaining was quantified according to the area percentages: 0 (no positive staining), $1(<10 \%), 2(10-50 \%)$ or $3(>50 \%)$. The sum of the extent and intensity scores was the final staining scores (0-6). Scores less than 3 were considered to indicate low expression, while scores $\geq 4$ were considered as high expression. All slides were examined by three experienced pathologists.

$R N A$ extraction and $R T-q P C R$. A total of $12 \mathrm{GC}$ tissue specimens were detected by RT-qPCR analysis. Total RNA from the tissue samples was extracted using TRIzol reagent (Takara Bio, Inc., Otsu, Japan). RNA samples (20 $\mu$ l) were reverse transcribed into cDNA using the Reverse Transcription kit (Takara Bio, Inc.), according to the manufacturer's protocol. RT-qPCR assays were performed using SYBR Green PCR master mix on a LightCycler 480 system (Roche Diagnostics, Basel,
Switzerland). The PCR cycling conditions were as follows: $95^{\circ} \mathrm{C}$ for $2 \mathrm{~min}$, followed by 40 cycles of $95^{\circ} \mathrm{C}$ for $30 \mathrm{sec}$ and $60^{\circ} \mathrm{C}$ for $35 \mathrm{sec}$. The FASN primers and probes (Invitrogen; Thermo Fisher Scientific, Inc., Waltham, MA, USA) were used for the amplification of a 20 base pair FASN-specific PCR product: Forward primer 5'-CGACAGCACCAGCTTCGC CA-3', reverse primer 5'-CACGCTGGCCTGCAGCTTCT-3'. Expression data were normalized to the housekeeping gene glyceraldehyde 3-phosphate dehydrogenase (GAPDH) to be calculated as $2^{-[(\mathrm{Cq} \text { of FASN)-(Cq of GAPDH)] }}$, where $\mathrm{Cq}$ represents the quantification cycle for each transcript (24).

Protein extraction and western blotting. A total of three GC tissues were lysed and subjected to western blotting as described previously (25). Briefly, the protein concentrations of the lysates were quantified using a Bicinchoninic Acid Assay kit (Nanjing KeyGen Biotech, Co., Ltd., Nanjing, China). Protein samples $(40 \mu \mathrm{g})$ were separated by $6 \%$ sodium dodecyl sulfate-polyacrylamide gel electrophoresis (Shanghai Shenggong Biology Engineering Technology Service, Ltd., Shanghai, China) and were then electrophoretically transferred to a polyvinylidene difluoride membrane (EMD Millipore, Billerica, MA, USA). Membranes were incubated in $5 \%$ bovine serum albumin (ZSGB-BIO, Beijing, China) in PBS for $1 \mathrm{~h}$, then with rabbit anti-human FASN polyclonal antibodies (1:1,000; 3180S; Cell Signaling Technology, Inc.), overnight at $4^{\circ} \mathrm{C}$. Membranes were then washed three times with PBS and incubated with HRP-conjugated goat anti-rabbit IgG (1:15,000; PV-6001; LI-COR, Inc.) for $1 \mathrm{~h}$ at room temperature. Antibody complexes were detected using enhanced chemiluminescence (PerkinElmer, Inc., Waltham, MA, USA) and the blots were scanned using the Odyssey CLx Imaging System (LI-COR, Inc.). The intensity of protein bands were quantified using Quantity One software, version 4.6.2 (Bio-Rad Laboratories, Inc., Hercules, CA, USA).

Statistical analysis. All statistical analyses were conducted using SPSS statistical software, version 20.0 (IBM SPSS, Armonk, NY, USA). Student's t-test analysis was performed to compare the statistical significance between two experimental groups. The association between FASN expression and clinicopathological characteristics was analyzed using the Chi-square test. Kaplan-Meier analyses were used to analyze the incident of patients (diagnosis of recurrence or mortality) and comparisons of survival distributions were evaluated with the log-rank test. A stepwise Cox's proportional hazard model was conducted to examine the univariate survival analyses. The quantitative data are represented as the mean \pm standard error of three independent experiments. $\mathrm{P}<0.05$ was considered to indicate a statistically significant difference.

\section{Results}

FASN was overexpressed in GC. The expression levels of FASN were assessed in $27 \mathrm{GC}$ tumor tissues and paired adjacent normal tissues (NT) by IHC. FASN was observed to be predominantly expressed in the cytoplasm (Fig. 1A) and infrequently in the nucleus (data not shown). Student's t-test, identified significantly greater expression of FASN in GC 
A

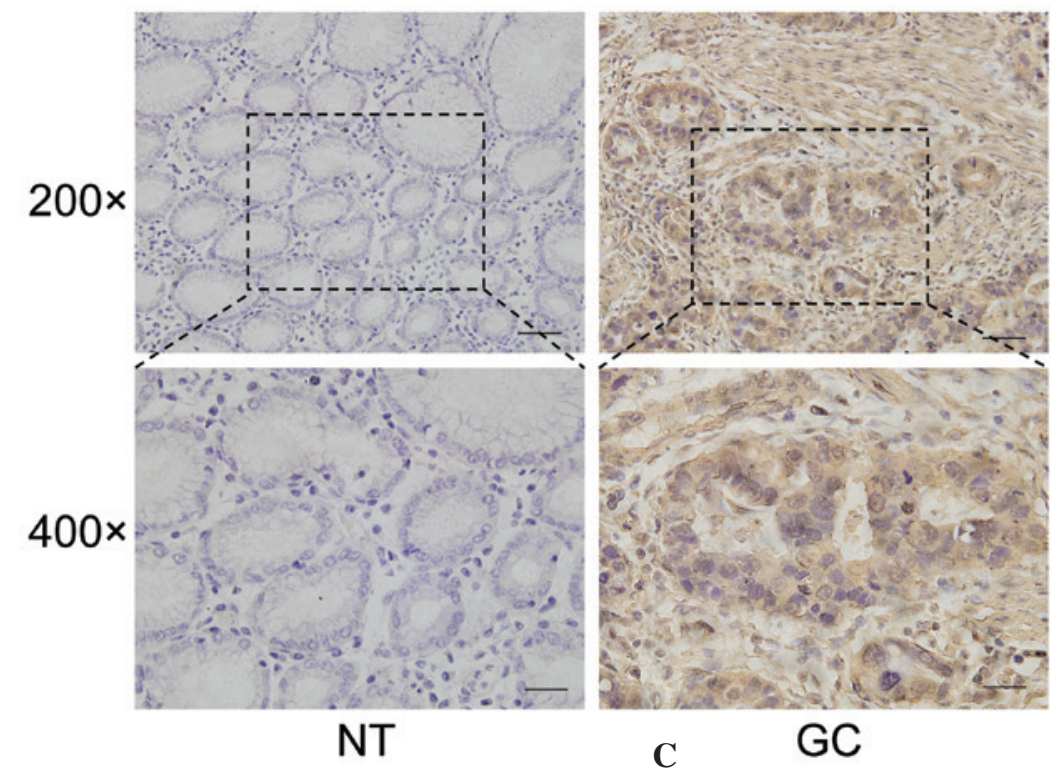

B

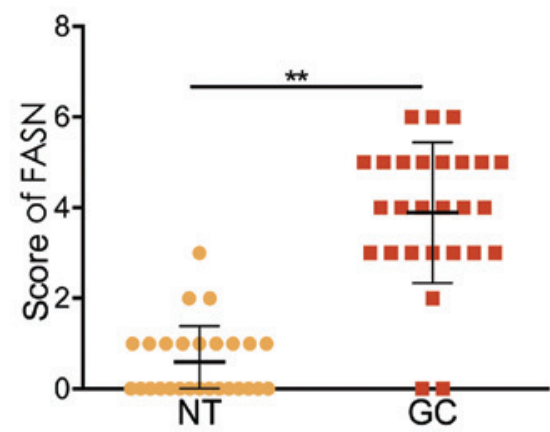

D

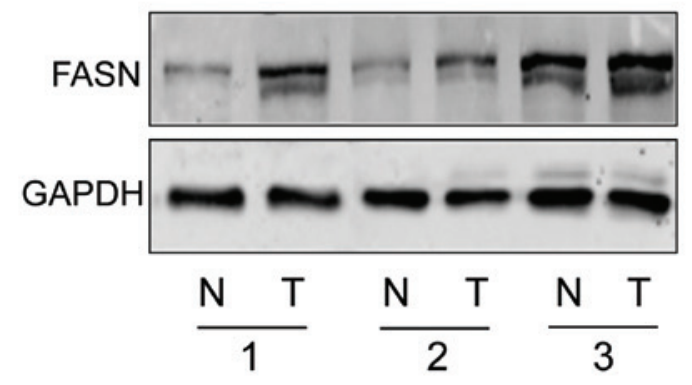

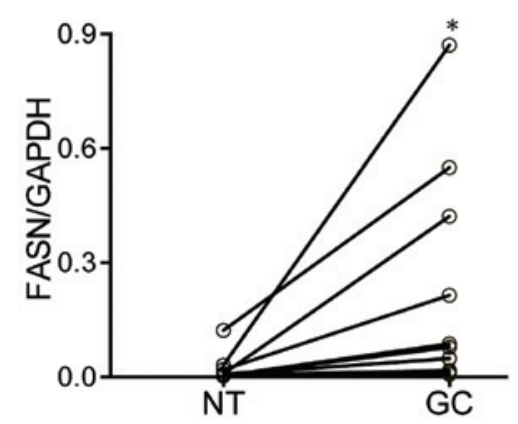

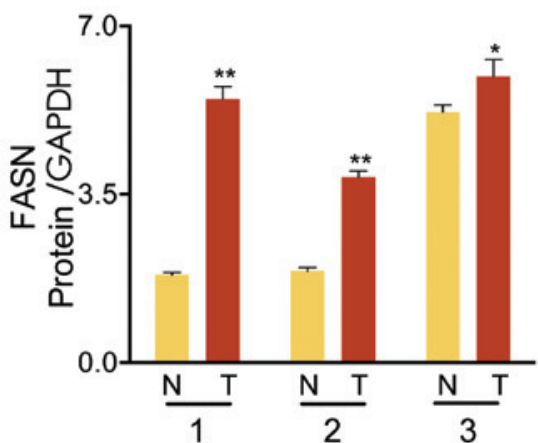

Figure 1.FASN was overexpressed in GC. (A) Representative immunohistochemistry analysis of FASN expression in the primary GC and adjacent NT samples. (B) Statistical quantification of FASN staining between GC and NT tissues. FASN expression was significantly higher in GC than that of NT. (C) Relative expression of FASN mRNA in 12 paired GC tissues and NT. (D) Western blotting of FASN in 3 paired GC and NT samples. FASN was weakly expressed in the normal tissues, however its expression was significantly increased in GC tissues ( $\mathrm{n}=3$ ). Left panel, western blotting; right panel, semi-quantitative assessment of FASN expression relative to the loading control, GAPDH. ${ }^{*} \mathrm{P}<0.05,{ }^{* *} \mathrm{P}<0.001$. Error bars represent the mean \pm standard deviation from three independent experiments. FASN, fatty acid synthase; GC, gastric cancer; NT, non-cancerous tissue; GAPDH, glyceraldehyde 3-phosphate dehydrogenase.

tissues when compared with paired adjacent normal tissues $(\mathrm{P}<0.001$, Fig. 1B). Similar results were also obtained from RT-qPCR (in 12 paired GC tissues and adjacent non-cancerous tissues; Fig. 1C) and western blot analysis (3 paired tissues; Fig. 1D). These results strongly indicated that FASN was overexpressed in GC tissues at mRNA and protein levels.

Expression of FASN was correlated with clinicopathological characteristics in $G C$. In order to determine the clinical significance of FASN, the association between FASN expression and the clinicopathological features of GC was investigated in a retrospective cohort of 167 cases of GC by IHC, including
19 cases of stage I (11.4\%), 49 cases of stage II (29.3\%), 63 cases of stage III (37.7\%) and 36 cases of stage IV (21.6\%), which were based on TNM staging. Among the tumors, 85 (50.9\%) samples presented with an overexpression of FASN (score >3), whereas $82(49.1 \%)$ cases were weakly or negatively expressed (scored 0-3).

Representative results of FASN staining are presented in Fig. 2A. Staining of FASN protein was observed to grow from weak to strong with the increased clinical stage of GC tissue specimens. Quantitative analysis of IHC staining indicated that FASN expression was positively correlated with clinical stage in primary GC tumors (Fig. 2B). The association between 
A

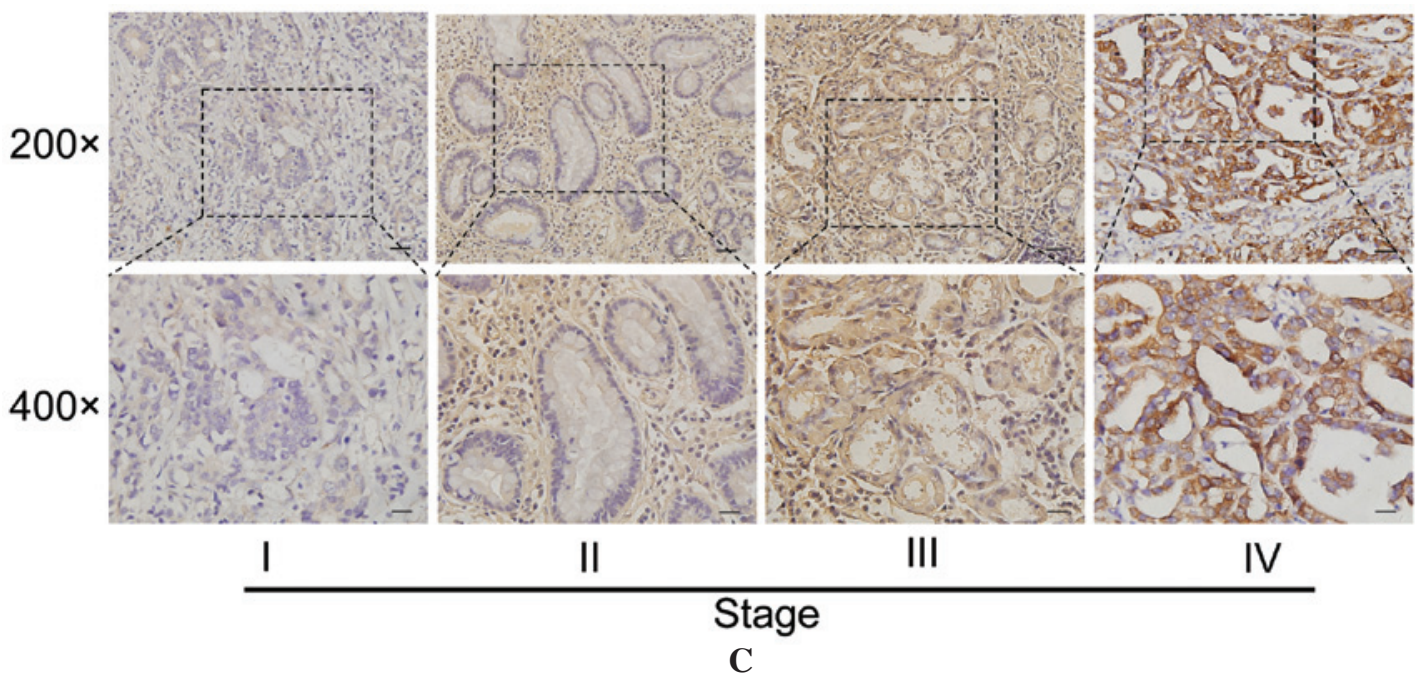

B
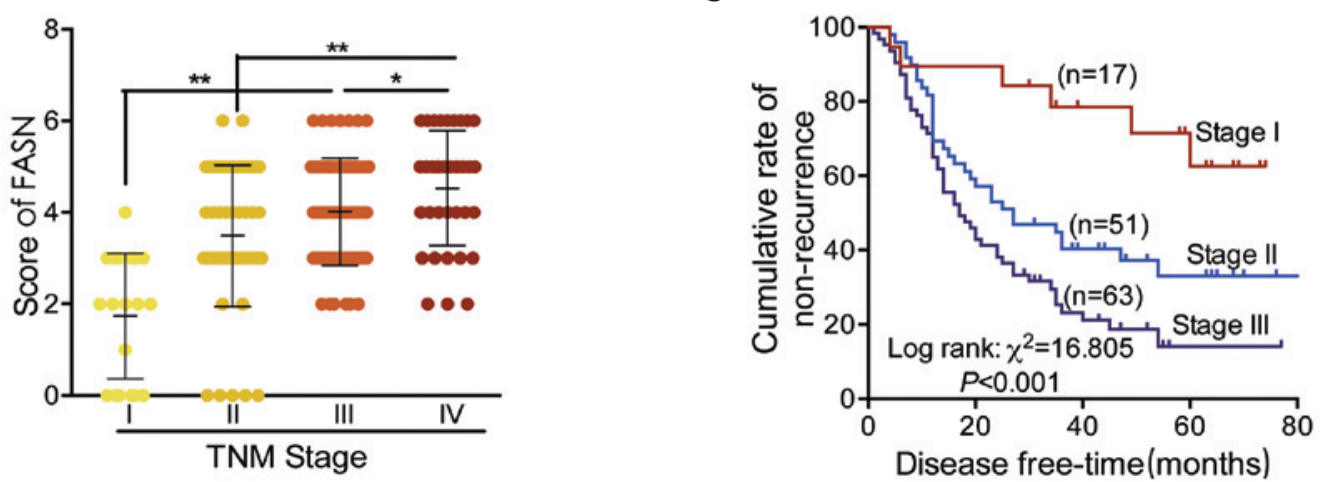

Figure 2. Overexpression of FASN in advanced GC. (A) Representative images of FASN protein immunostaining in different TNM stages of GC tissue. (B) Staining scores for FASN in each group. (C) Kaplan-Meier curves for postoperative recurrence of stages I-III GC in relation to disease stage. * $<<0.05$, ${ }^{* *} \mathrm{P}<0.001$. FASN, fatty acid synthase; GC, gastric cancer; TNM, tumor, nodes, metastasis.

the clinical stages and prognosis in patients with GC was additionally measured using survival analysis (Fig. 2C). As hypothesized, patients in stage III were identified to have the lowest non-recurrent rate $(20.6 \%)$, while patients in stage I presented with the best outcome, with a $68.4 \%$ non-recurrent rate.

Clinical features were summarized in Table I. In patients from stages I-III, expression of FASN was observed to be positively correlated with age $(\mathrm{P}<0.05)$, TNM classification $(\mathrm{P}<0.001)$, gastric wall invasion $(\mathrm{P}=0.014)$, nodal metastasis $(\mathrm{P}<0.001)$ and postoperative recurrence (including local recurrence and distant metastasis; $\mathrm{P}<0.001)$. For patients in stage IV, FASN overexpression was closely associated with the number of metastatic lesions $(\mathrm{P}<0.001)$ and mortality $(\mathrm{P}=0.001)$. Taken together, these results indicate that FASN expression had a strong correlation with GC invasion, metastasis and prognosis.

FASN overexpression was positively associated with the number of metastatic lesions in GC. To further explore the association between FASN expression and metastasis, IHC analyses were conducted. Consequently, FASN was significantly overexpressed in tissues with multiple metastatic lesions (MML) (>3), compared with less metastatic lesions (LML) $(\leq 3)$ in stage IV patients $(\mathrm{P}<0.05$; Fig. $3 \mathrm{~A}$ and $\mathrm{B})$. Besides, the Kaplan-Meier survival curve further demonstrated that patients with MML had a lower median survival time of 7.7 months, compared with 24 months in LML (log-rank
$\mathrm{P}<0.05$, Fig. 3C). These highly indicated an important role for FASN in GC metastasis.

FASN overexpression predicted a poor prognosis of $G C$. To identify the potential prognostic role of FASN in GC, multiple analyses were conducted. Firstly, FASN expression was examined in patients with stage I-III GC with recurrence/non-recurrence in addition to stage IV patients with survival/mortality by IHC staining. The results demonstrated that FASN was significantly overexpressed in patients with recurrence $(\mathrm{P}<0.05$; Fig. $4 \mathrm{~A})$ or mortality $(\mathrm{P}<0.05$; Fig. 4B).

Secondly, Kaplan-Meier analyses were also performed. As presented in Fig. 4C, the data suggested that the FASN levels were significantly associated with postoperative recurrence in patients with stage I-III GC. FASN low-expression was associated with a non-recurrent rate of $39.2 \%$, in contrast with $26.3 \%$ for the FASN high-expression group. The median time to recurrence was 35 months in patients with stages I-III GC with low-expression of FASN vs. 14 months in those with overexpression. In patients with stage IV GC, low-expression of FASN resulted in a 5-year survival rate of 50.0\%, which was reduced to $32.1 \%$ in the FASN-overexpressed group. The median survival time of stage IV patients with FASN overexpression was 9.5 months vs. 35 months for those with low-expression (Fig. 4D).

In Cox's regression model, clinical stage $(\mathrm{P}<0.001 ; \mathrm{HR}$, 1.931; 95\% CI, 1.392-2.680) was an independent factor in 
Table I. Clinicopathological characteristics in response to positive and negative gastric FASN immunohistochemical staining.

A, Recurrence in stage I-III GC

\begin{tabular}{|c|c|c|c|c|}
\hline \multirow[b]{2}{*}{ Characteristic } & \multirow[b]{2}{*}{$\mathrm{n}(\%)$} & \multicolumn{2}{|c|}{ FASN expression } & \multirow[b]{2}{*}{ P-value } \\
\hline & & Positive n (\%) & Negative n (\%) & \\
\hline Gender & & & & 0.4 \\
\hline Male & $88(67.2)$ & $37(42.0)$ & $51(58.0)$ & \\
\hline Female & $43(32.8)$ & $20(46.5)$ & $23(53.5)$ & \\
\hline Age (years) & & & & 0.032 \\
\hline$\geq 55$ & $77(58.8)$ & $40(51.9)$ & $37(48.1)$ & \\
\hline$<55$ & $54(41.2)$ & $17(31.5)$ & $37(68.5)$ & \\
\hline Stage of disease & & & & $<0.001$ \\
\hline I & $19(14.5)$ & $1(5.3)$ & $18(94.7)$ & \\
\hline II & $49(37.4)$ & $16(32.7)$ & $33(67.3)$ & \\
\hline III & $63(48.1)$ & $40(63.5)$ & $23(36.5)$ & \\
\hline Gastric wall invasion & & & & 0.014 \\
\hline $\mathrm{T} 1-3$ & $76(58.0)$ & $26(34.2)$ & $50(65.8)$ & \\
\hline $\mathrm{T} 4$ & $55(42.0)$ & $31(56.4)$ & $24(43.6)$ & \\
\hline Nodal metastasis & & & & $<0.001$ \\
\hline Negative & $49(37.4)$ & $12(24.5)$ & $37(75.5)$ & \\
\hline Positive & $82(62.6)$ & $45(54.9)$ & $37(45.1)$ & \\
\hline Recurrence & & & & $<0.001$ \\
\hline No & $44(33.6)$ & $15(34.1)$ & $29(65.9)$ & \\
\hline Yes & $87(66.4)$ & $42(48.3)$ & $45(51.7)$ & \\
\hline
\end{tabular}

B, Survival in stage IV GC

FASN expression

\begin{tabular}{lccc} 
Characteristic & $\mathrm{n}(\%)$ & Positive $\mathrm{n}(\%)$ & Negative n $(\%)$ \\
\hline Gender & & & $4(16.7)$ \\
$\quad$ Male & $24(66.7)$ & $8(63.3)$ & $4(33.3)$ \\
Female & $12(33.3)$ & & 0.182 \\
Age (years) & & $21(87.3)$ & $5(12.7)$ \\
$\geq 55$ & $26(72.2)$ & $7(70.0)$ & $3(30.0)$ \\
$<55$ & $10(27.8)$ & $11(64.7)$ & $6(35.3)$ \\
Metastatic lesions & & $17(89.5)$ & $2(10.5)$ \\
$<3$ & $17(47.2)$ & & $4(44.4)$ \\
$\geq 3$ & $19(52.8)$ & $5(55.6)$ & $4(14.8)$ \\
Survival & $9(25.0)$ & $23(85.2)$ & $<0.001$ \\
No & $27(75.0)$ & & 0.001 \\
Yes & & & \\
\hline
\end{tabular}

FASN, fatty acid synthase; GC, gastric cancer.

predicting the risk of recurrence following radical surgery in patients with stage I-III GC, and the number of metastatic lesions ( $\mathrm{P}=0.018$; HR, 2.676; 95\% CI, 1.184-6.049) was also considered as an independent predictor of mortality for patients with stage IV GC. More importantly, compared with patients with low-expression of FASN, those with FASN overexpression were observed to exhibit a significant increase in GC-postoperative recurrence rate $(\mathrm{P}=0.014$; HR, $1.705 ; 95 \%$ CI, 1.116-2.606), in addition to overall mortality $(\mathrm{P}=0.008$; HR, 4.412; 95\% CI, 1.463-13.305; Table II). Taken 
A

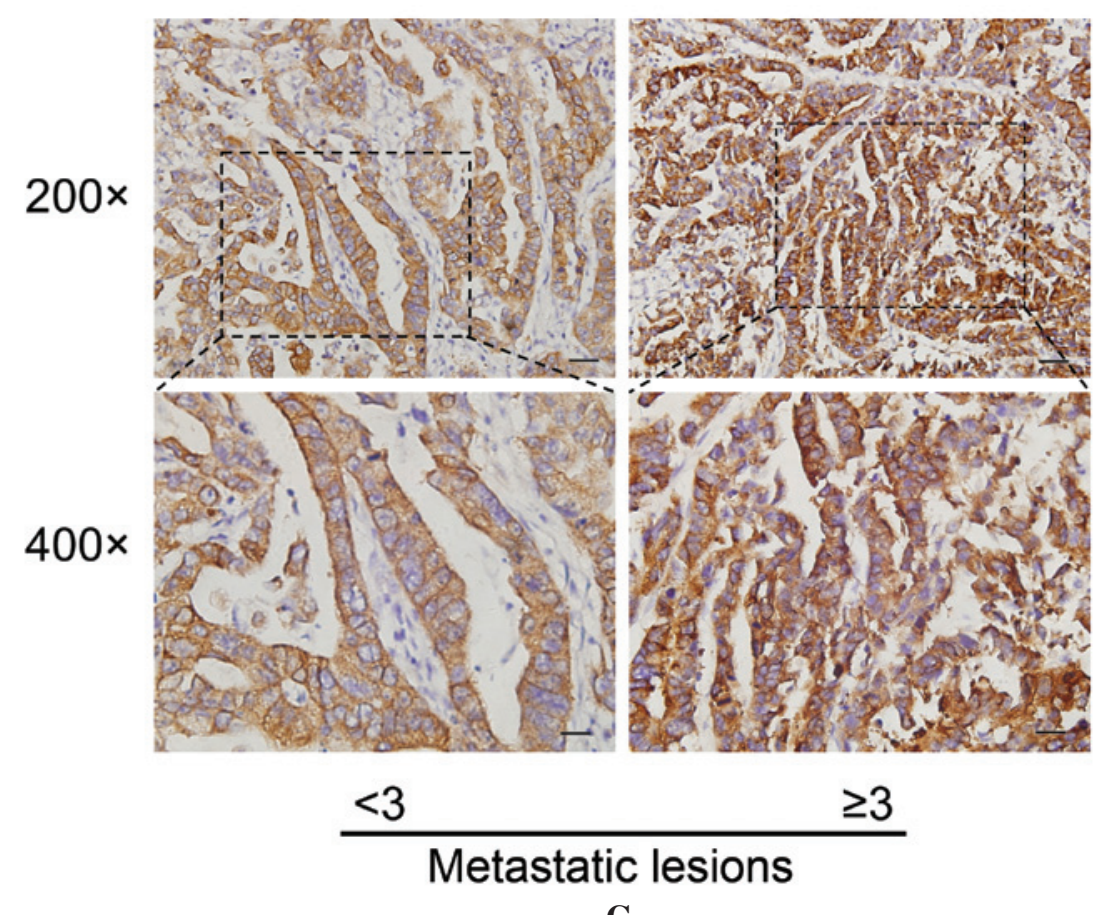

B

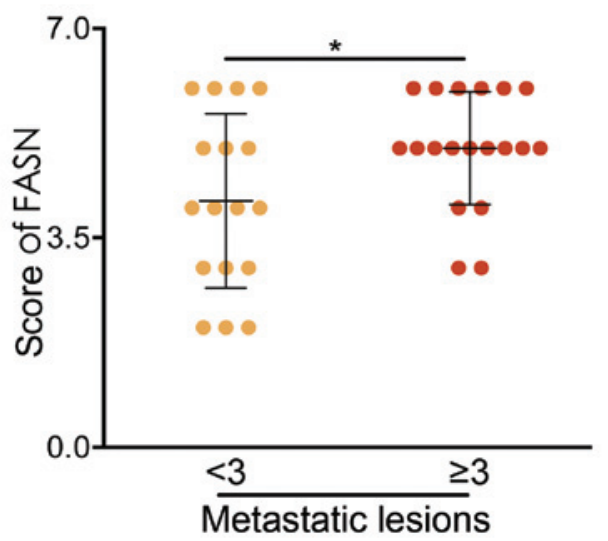

$\mathrm{C}$

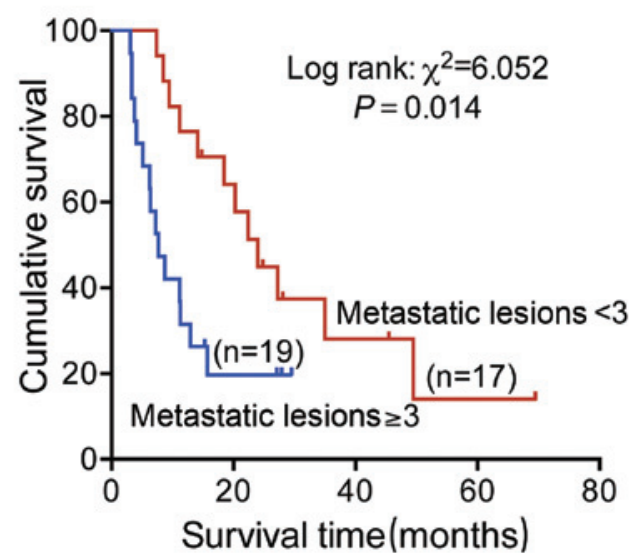

Figure 3. FASN overexpression was positively associated with the number of metastatic lesions in gastric cancer. (A) Representative images of FASN protein immunostaining in tissues with multiple metastatic lesions ( $>3)$ and less metastatic lesions $(\leq 3)$ in stage IV patients. (B) Staining scores for FASN in each group, ${ }^{*} \mathrm{P}<0.05$. (C) Survival of stage-IV patients analyzed in terms of the number of metastatic lesions. ${ }^{*} \mathrm{P}=0.014$. FASN, fatty acid synthase.

together, the results suggested a potentially promising prognostic value of FASN for patients with GC.

\section{Discussion}

Tumor metabolism is a key process in cancer growth and progression. Numerous studies have demonstrated that the glucose metabolism is essential in cancer cell growth and invasion. However, few studies have focussed upon the role of lipid metabolism in tumor development (26). Thus, the current study investigated the potential role of the lipid metabolism in GC.

FASN is a key enzyme in the lipid metabolism, the expression of which has clear potential in aiding in the understanding of the lipid metabolism. FASN has been previously identified to be overexpressed in several types of solid tumors and associated with poor prognosis of urothelial carcinoma (27), pancreatic neoplasia (28) and renal cell carcinoma (29). These studies proposed a crucial role for FASN in tumor progression, however, reports concerning FASN in GC remain rare. Hou et al (21) identified that FASN overexpression was significantly correlated with poor prognosis in $90 \mathrm{GC}$ specimens. In addition, Ito et al (22) observed that FASN was detected in the serum by enzyme-linked immunosorbent assay in patients with GC and normal controls. In addition, they observed that FASN was highly expressed in the serum of patients with GC compared with those of healthy people. In the present study, the IHC staining experiment was repeated in a larger GC sample size, and the association between FASN and clinicopathological parameters including age, gender, gastric wall invasion, recurrence in stage I-III patients and survival time in stage IV patients was investigated. Consequently, similar results were obtained, identifying that expression of FASN was higher in GC tissues than those in adjacent non-cancerous tissues, and FASN was significantly associated with poor prognosis of GC, indicating that FASN overexpression contributed to GC development.

Notably, previous studies demonstrated that FASN was not significantly associated with nodal metastasis $(21,22)$. 
Table II. Hazard analysis for recurrence incidence and overall survival rate.

$95 \%$ CI for HR

HR

Covariates

P-value

(Hazard ratio)

1.097

0.702

0.717

1.392

1.931

1.228

$<0.001$

Gastric wall invasion

Nodal metastasis (vs. No)

FASN expression (vs. No)

0.004

0.014

1.969

1.705

0.351

1.490

0.439

0.018

0.008
Age (vs. <55 years)

Metastatic lesions (vs. <3)

FASN expression (vs. no)
1.439

0.804

1.237

1.116

CI, confidence interval; HR, hazard ratio; GC, gastric cancer.

A

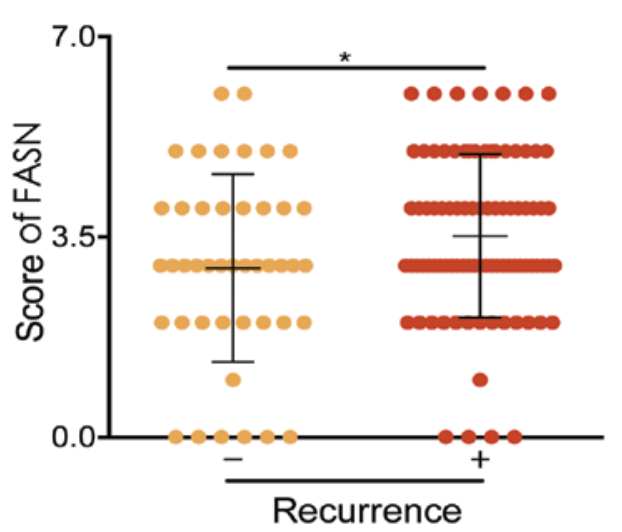

C

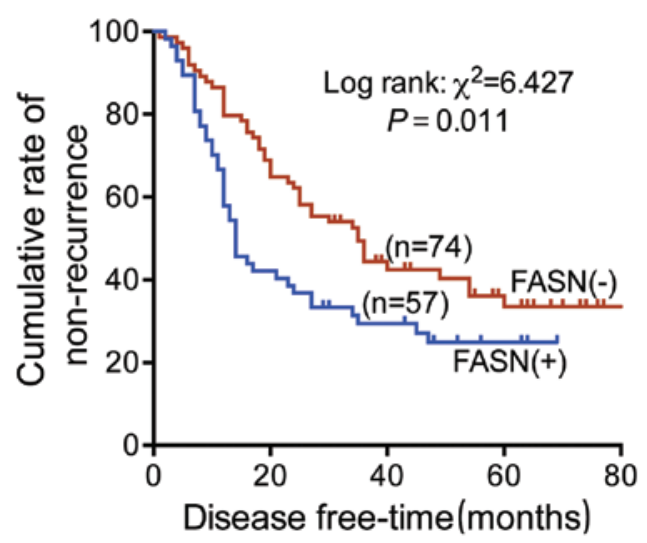

B

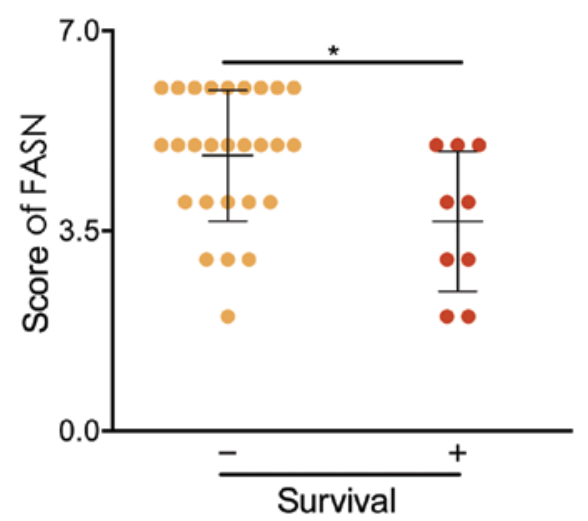

D

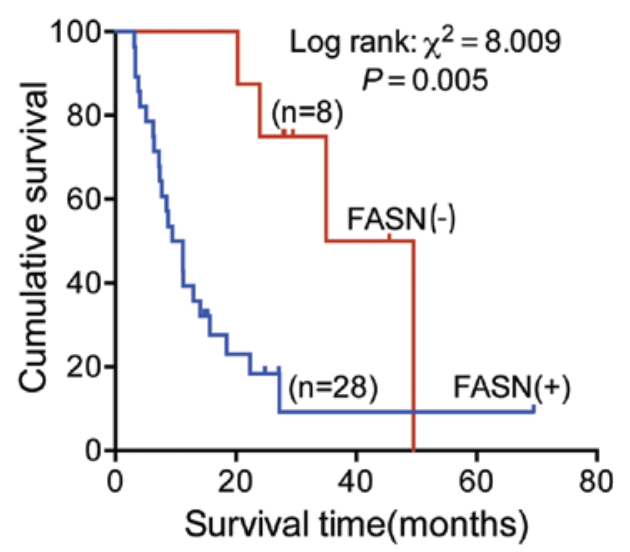

Figure 4. FASN overexpression predicted a poor prognosis of GC. Staining scores for (A) FASN in postoperative recurrence of stages I-III patients with GC and (B) the mortality of stage IV patients with GC. (C) Kaplan-Meier curves for postoperative recurrence of stages I-III GC in relation to FASN expression. (D) Survival of stage IV patients analyzed in relation to FASN expression. FASN, fatty acid synthase; GC, gastric cancer.

However, in the present study, the analysis demonstrated that FASN was positively associated with lymph node metastasis. The differences in the sample size and the individuals may contribute to this discrepancy. FASN has however been previously observed to be associated with nodal metastasis in several other types of tumor (30-32), thus suggesting that 
the results are reliable, and suggesting a potential role of FASN in the early detection and lymphangiogenesis of GC. In addition, the current study was, to the best of our knowledge, the first to provide evidence that FASN was associated with the number of tumor metastatic lesions, thus indicating that FASN contributes to the development and progression of GC by promoting invasion and metastasis of GC cells. Several previous studies have identified that FASN overexpression promotes proliferation, invasion and metastasis in certain types of cancer $(18,33,34)$. By conducting RT-qPCR and western blotting, the mRNA and protein expression levels of FASN were detected in GC tissues, thus providing evidence that FASN contributes to GC progression at the transcriptional and translational levels. However, the underlying mechanisms require further investigation. The results of the present study suggest that FASN may serve as a novel prognostic marker of GC. Identification of FASN as a prognostic biomarker for $\mathrm{GC}$ in the current study provides insight into the mechanisms associated with poor prognosis.

In summary, by analyzing the expression of GC tissues and adjacent non-cancerous tissues, the current study demonstrated that FASN may act as a novel prognostic marker. Furthermore, lipid metabolism disorders were identified to participate in the development and progression of GC.

\section{Acknowledgements}

The present study was supported by the National Natural Science Foundation of China (grant no. 81472314 to Professor Wangjun Liao; grant no. 81472317 to Professor Shi Min) and the Special Foundation for National Clinical Specialties of China (to the Department of Oncology, Nanfang Hospital, Southern Medical University, Guangzhou, China).

\section{References}

1. Siegel R, Ma J, Zou Z and Jemal A: Cancer statistics, 2014. CA Cancer J Clin 64: 9-29, 2014.

2. Jemal A, Bray F, Center MM, Ferlay J, Ward E and Forman D: Global cancer statistics. CA Cancer J Clin 61: 69-90, 2011.

3. Bertuccio P, Chatenoud L, Levi F, Praud D, Ferlay J, Negri E, Malvezzi M and La Vecchia C: Recent patterns in gastric cancer: A global overview. Int J Cancer 125: 666-673, 2009.

4. Blum MA, Takashi T, Suzuki A and Ajani JA: Management of localized gastric cancer. J Surg Oncol 107: 265-270, 2013.

5. Rios P, Maruyama K, Sasako M, Sano T and Katai H: The current situation and treatment strategy for gastric cancer at the national cancer center of Tokyo, Japan. Rev Gastroenterol Peru 14: 197-203, 1994 (In Spanish).

6. Cidon EU, Ellis SG, Inam Y, Adeleke S, Zarif S and Geldart T: Molecular targeted agents for gastric cancer: A step forward towards personalized therapy. Cancers (Basel) 5: 64-91, 2013.

7. Varis A, Zaika A, Puolakkainen P, Nagy B, Madrigal I, Kokkola A, Väyrynen A, Kärkkäinen P, Moskaluk C, El-Rifai W and Knuutila S: Coamplified and overexpressed genes at ERBB2 locus in gastric cancer. Int J Cancer 109: 548-553, 2004

8. Califano D, Pignata S, Losito NS, Ottaiano A, Greggi S, De Simone V, Cecere S, Aiello C, Esposito F, Fusco A and Chiappetta G: High HMGA2 expression and high body mass index negatively affect the prognosis of patients with ovarian cancer. J Cell Physiol 229: 53-59, 2014.

9. Bi X, Rexer B, Arteaga CL, Guo M and Mahadevan-Jansen A: Evaluating HER2 amplification status and acquired drug resistance in breast cancer cells using Raman spectroscopy. J Biomed Opt 19: 025001, 2014.
10. Li W, Tai Y, Zhou J, Gu W, Bai Z, Zhou T, Zhong Z, McCue PA, Sang N, Ji JY, et al: Repression of endometrial tumor growth by targeting SREBP1 and lipogenesis. Cell Cycle 11: 2348-2358, 2012.

11. Koochekpour S, Majumdar S, Azabdaftari G, Attwood K, Scioneaux R, Subramani D, Manhardt C, Lorusso GD, Willard SS, Thompson H, et al: Serum glutamate levels correlate with Gleason score and glutamate blockade decreases proliferation, migration, and invasion and induces apoptosis in prostate cancer cells. Clin Cancer Res 18: 5888-5901, 2012.

12. Zadra G, Photopoulos C, Tyekucheva S, Heidari P, Weng QP, Fedele G, Liu H, Scaglia N, Priolo C, Sicinska E, et al: A novel direct activator of AMPK inhibits prostate cancer growth by blocking lipogenesis. EMBO Mol Med 6: 519-538, 2014.

13. Yang CS, Matsuura K, Huang NJ, Robeson AC, Huang B, Zhang L and Kornbluth S: Fatty acid synthase inhibition engages a novel caspase-2 regulatory mechanism to induce ovarian cancer cell death. Oncogene 34: 3264-3272, 2015.

14. Jiang L, Xiao L, Sugiura H, Huang X, Ali A, Kuro OM, Deberardinis RJ and Boothman DA: Metabolic reprogramming during TGF 31 -induced epithelial-to-mesenchymal transition. Oncogene 34: 3908-3916, 2015.

15. Ogino S, Nosho K, Meyerhardt JA, Kirkner GJ, Chan AT, Kawasaki T, Giovannucci EL, Loda M and Fuchs CS: Cohort study of fatty acid synthase expression and patient survival in colon cancer. J Clin Oncol 26: 5713-5720, 2008.

16. Li J, Dong L, Wei D, Wang X, Zhang S and Li H: Fatty acid synthase mediates the epithelial-mesenchymal transition of breast cancer cells. Int J Biol Sci 10: 171-180, 2014.

17. Grube S, Dünisch P, Freitag D, Klausnitzer M, Sakr Y, Walter J, Kalff R and Ewald C: Overexpression of fatty acid synthase in human gliomas correlates with the WHO tumor grade and inhibition with Orlistat reduces cell viability and triggers apoptosis. J Neurooncol 118: 277-287, 2014.

18. Agostini M, Almeida LY, Bastos DC, Ortega RM, Moreira FS, Seguin F, Zecchin KG, Raposo HF, Oliveira HC, Amoêdo ND, et al: The fatty acid synthase inhibitor orlistat reduces the growth and metastasis of orthotopic tongue oral squamous cell carcinomas. Mol Cancer Ther 13: 585-595, 2014.

19. Zaytseva YY, Rychahou PG, Gulhati P, Elliott VA, Mustain WC, O'Connor K, Morris AJ, Sunkara M, Weiss HL, Lee EY and Evers BM: Inhibition of fatty acid synthase attenuates CD44-associated signaling and reduces metastasis in colorectal cancer. Cancer Res 72: 1504-1517, 2012.

20. Tomek K, Wagner R, Varga F, Singer CF, Karlic H and Grunt TW: Blockade of fatty acid synthase induces ubiquitination and degradation of phosphoinositide-3-kinase signaling proteins in ovarian cancer. Mol Cancer Res 9: 1767-1779, 2011.

21. Hou W, Fei M, Qin X, Zhu X, Greshock J, Liu P, Zhou Y, Wang H, Ye BC and Qin CY: High overexpression of fatty acid synthase is associated with poor survival in Chinese patients with gastric carcinoma. Exp Ther Med 4: 999-1004, 2012.

22. Ito T, Sato K, Maekawa H, Sakurada M, Orita H, Shimada K, Daida H, Wada R, Abe M, Hino O and Kajiyama Y: Elevated levels of serum fatty acid synthase in patients with gastric carcinoma. Oncol Lett 7: 616-620, 2014.

23. Edge SB and Compton CC: The American Joint Committee on Cancer: The 7th edition of the AJCC cancer staging manual and the future of TNM. Ann Surg Oncol 17: 1471-1474, 2010.

24. Livak KJ and Schmittgen TD: Analysis of relative gene expression data using real-time quantitative PCR and the $2-\Delta \Delta \mathrm{Ct}$ method. Methods 25: 402-408, 2001

25. Wang L, Wu Y, Lin L, Liu P, Huang H, Liao W, Zheng D, Zuo Q, Sun L and Huang N: Metastasis-associated in colon cancer-1 upregulation predicts a poor prognosis of gastric cancer, and promotes tumor cell proliferation and invasion. Int J Cancer 133: 1419-1430, 2013.

26. Swierczynski J, Hebanowska A and Sledzinski T: Role of abnormal lipid metabolism in development, progression, diagnosis and therapy of pancreatic cancer. World J Gastroenterol 20: 2279-2303, 2014.

27. Hamada S, Horiguchi A, Asano T, Kuroda K, Asakuma J, Ito K, Asano T, Miyai K and Iwaya K: Prognostic impact of fatty acid synthase expression in upper urinary tract urothelial carcinoma. Jpn J Clin Oncol 44: 486-492, 2014.

28. Walter K, Hong SM, Nyhan S, Canto M, Fedarko N, Klein A, Griffith M, Omura N, Medghalchi S, Kuhajda F and Goggins M: Serum fatty acid synthase as a marker of pancreatic neoplasia. Cancer Epidemiol Biomarkers Prev 18: 2380-2385, 2009. 
29. Horiguchi A, Asano T, Asano T, Ito K, Sumitomo M and Hayakawa M: Fatty acid synthase over expression is an indicator of tumor aggressiveness and poor prognosis in renal cell carcinoma. J Urol 180: 1137-1140, 2008.

30. Long QQ, Yi YX, Qiu J, Xu CJ and Huang PL: Fatty acid synthase (FASN) levels in serum of colorectal cancer patients: Correlation with clinical outcomes. Tumour Biol 35: 3855-3859, 2014.

31. Silva SD, Perez DE, Nishimoto IN, Alves FA, Pinto CA, Kowalski LP and Graner E: Fatty acid synthase expression in squamous cell carcinoma of the tongue: Clinicopathological findings. Oral Dis 14: 376-382, 2008.
32. Tsuji T, Yoshinaga M, Togami S, Douchi T and Nagata Y: Fatty acid synthase expression and clinicopathological findings in endometrial cancer. Acta Obstet Gynecol Scand 83: 586-590, 2004.

33. Zaytseva YY, Elliott VA, Rychahou P, Mustain WC, Kim JT, Valentino J, Gao T, O'Connor KL, Neltner JM, Lee EY, et al: Cancer cell-associated fatty acid synthase activates endothelial cells and promotes angiogenesis in colorectal cancer. Carcinogenesis 35: 1341-1351, 2014.

34. Li N, Bu X, Tian X, Wu P, Yang L and Huang P: Fatty acid synthase regulates proliferation and migration of colorectal cancer cells via HER2-PI3K/Akt signaling pathway. Nutr Cancer 64: 864-870, 2012. 